\title{
Perfil de trabalhadores com doenças da coluna vertebral atendidos em um serviço de saúde
}

\author{
Janaina Bussola Montrezor Valença ${ }^{a}$, Karoline Pereira Ferraza , Maria do Carmo Baracho de Alencar ${ }^{2}$, \\ Felipe Granado Souza ${ }^{a}$, Lucy Vitale Lopes ${ }^{b}$ \\ ${ }^{a}$ Universidade Federal de São Paulo - UNIFESP, Santos, SP, Brasil.

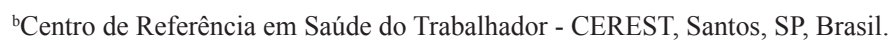

\begin{abstract}
Resumo: Objetivo: Descrever o perfil de trabalhadores com doenças da coluna vertebral (dorsopatias) atendidos em um serviço de Saúde do Trabalhador. Método: Estudo quantitativo com análise documental de prontuários abertos de pacientes atendidos no Centro de Referência em Saúde do Trabalhador (CEREST), Santos-SP, de janeiro de 2010 a dezembro de 2011, e seleção de prontuários de sujeitos com diagnóstico clínico conforme a Classificação Internacional de Doenças CID-10 de M50 a M54. Resultados: Foram analisados 431 prontuários e 15,7\% (n=68) apresentaram diagnóstico pela CID-10 de M50 a M54, de pacientes de ambos os gêneros, faixa etária mais acometida entre 41 e 50 anos. A principal categoria acometida foi 'outras atividades de serviços', com 24,6\% ( $\mathrm{n}=16)$. Das análises, 27,9\% ( $\mathrm{n}=19)$ apresentaram também diagnóstico de transtornos dos tecidos moles (M60 a M79), 3,2\% $(n=09)$ apresentaram transtornos mentais, e 70,9\% (n=39) se encontravam em situação de afastamento do trabalho. Conclusão: A faixa etária mais acometida se encontrava em fase produtiva, mais da metade dos sujeitos estavam em situação de afastamento do trabalho e alguns sujeitos apresentaram outros diagnósticos clínicos, reforçando a necessidade de intervenções interdisciplinares.
\end{abstract}

Palavras-chave: Doenças da Coluna Vertebral, Dor Lombar, Saúde do Trabalhador, Terapia Ocupacional.

\section{Profile of workers with spine cord diseases attended in an occupational health service}

\begin{abstract}
Aim: To describe the profile of workers with spine cord diseases attended in an Occupational Health Service. Method: A quantitative study with documentary analysis of open records of patients attended at the Occupational Health Reference Service (CEREST), in Santos-SP, and record selection of subject with clinical diagnosis of M50 to M54 according to the ICD-10. Results: We analyzed 431 records of patients from both genders, most affected age between 41 and 50 years, and $15.7 \%(n=68)$ were diagnosed with M50 to M54 according to the ICD-10. The most affected professional category was 'other activities services', with $24.6 \%(\mathrm{n}=16)$. The analyses showed that $27.9 \%(n=19)$ presented also soft tissue injuries (M60 to M79), 3.2\% ( $n=09)$ mental disorders, and $70.9 \%(n=39)$ were in absence from work. Conclusion: The most affected age was in the productive phase and more than half of the subjects were in absence from work situation, some subjects with other clinical diagnosis, reinforcing the need of interdisciplinary interventions.
\end{abstract}

Keywords: Spinal Diseases, Low Back Pain, Occupational Health, Occupational Therapy. 


\section{Introdução}

Entre as doenças relacionadas ao trabalho, estão as dorsopatias (doenças da coluna vertebral), em que sintomas de dor (algia) são frequentes. Segundo a Classificação Internacional de Doenças (CID-10), para dor nas costas encontram-se as cervicalgias, dores torácicas, ciáticas, transtornos dos discos intervertebrais, espondiloses, radiculopatias, além das dores lombares (lombalgias), que são as mais comuns (HAGEN; TAMBS; BJERKEDAL, 2002). Conforme o Anuário Estatístico de Acidentes de Trabalho da Previdência Social referente ao ano de 2012, foram registrados 47.554 casos de Dorsalgias (M.54), de acordo com a CID-10, ocupando o segundo lugar de prevalência (BRASIL, 2012). A dor nas costas é um problema de saúde pública em vários países e com prejuízo econômico para a sociedade (EKMAN; JOHNELL; LIDGREN, 2005).

A dor nas costas decorre de um conjunto de causas, e não somente de doenças específicas, como fatores sociodemográficos (idade, gênero, renda e escolaridade), comportamentais (fumo e baixa atividade física), exposiçóes ocorridas nas atividades cotidianas (trabalho físico extenuante, vibração, posição viciosa, movimentos repetitivos) e outros, como a obesidade e morbidades psicológicas (FERREIRA et al., 2011). Nos acidentes e doenças do trabalho, a regiáo lombar é comumente acometida. Em estudo de Campbell et al. (2013), as doenças da coluna lombar foram muito comuns na população trabalhadora e representaram entre $60 \%$ e $80 \%$ das doenças.

O fator idade é apontado como risco para as lombalgias, especialmente nas faixas etárias acima de 40 anos e em fase produtiva (HEDEAGER MOMSEN et al., 2014; PEDROSO et al., 2013; HELFENSTEIN JUNIOR; GOLDEFUM; SIENA, 2010). Tanto os esforços dinâmicos relacionados a deslocamentos, a transporte de cargas, quanto os esforços estáticos relacionados com a sustentação de cargas pesadas, com a adoção de posturas incômodas, podem contribuir para as lesóes nas articulaçóes e nos discos intervertebrais (HELFENSTEIN JUNIOR; GOLDEFUM; SIENA, 2010).

As doenças da coluna lombar são qualificadas como entidades deficitárias musculoesqueléticas, caracterizadas por dor e limitação funcional, com a dor lombar afetando de 60 a $70 \%$ da população pelo menos uma vez durante a vida, e 10 a $15 \%$ dessa população desenvolve dor lombar crônica (PEDROSO et al., 2013). Os autores também citam que os desarranjos mecânico-posturais são fatores frequentemente associados à dor lombar, pois as cargas impostas aos discos, articulaçóes e ligamentos vertebrais por longos períodos de tempo podem estar relacionadas ao desencadeamento da dor. Para Leal (2010), há características individuais (como idade superior a 40 anos, gênero feminino e fatores genéticos), bem como fatores relacionados a condiçôes de trabalho (trabalho físico pesado, ritmo intenso, tarefa monótona, levantamento de pesos, posturas inadequadas, insatisfação, tarefa de baixo reconhecimento, baixo nível de decisão, estresse no trabalho, etc.) e ao estilo de vida (obesidade, tabagismo, sedentarismo, etc.), que aumentam o risco para a lombalgia.

Abreu e Ribeiro (2010) citam que atividades profissionais com grande sobrecarga física, somadas a uma postura inadequada, movimentos repetitivos, vibração, trabalhos em grande velocidade e estresse, reforçam o aparecimento dessa morbidade, expondo o trabalhador à dor, apresentando, portanto, um caráter ocupacional. O número de dias de trabalho perdidos por beneficiário em 2007 sugeriu que a invalidez por dor nas costas ocorresse mais cedo em alguns ramos de atividades (MEZIAT FILHO; AZEVEDO E SILVA, 2011). Autores trazem as incidências de lombalgias em várias atividades profissionais, como, por exemplo, em trabalhadores dos correios em atividades de entrega (MASCARENHA; BARBOSA-BRANCO, 2014); em motoristas de ônibus, mecânicos e operadores (ABREU; RIBEIRO, 2010); policiais militares (TAVARES NETO et al., 2013); costureiras (CARDOSO; BATISTA; SANDOVAL, 2011); e em trabalhadores de enfermagem (enfermeiros, técnicos e auxiliares de enfermagem) (LIMA et al., 2013); entre outras profissōes.

Almeida et al. (2008) dizem que a lombalgia é um sintoma altamente incapacitante que gera elevados níveis de absenteísmo no trabalho e elevados gastos com a saúde. A dor pode se tornar crônica, incapacitando os indivíduos para a realização de atividades profissionais, sociais e familiares (ALENCAR; TERADA, 2012). A procura por tratamento das dores lombares aumenta a cada dia, e a demanda em hospitais e clínicas ocasiona um aumento no custo de despesas em cuidados com a saúde, que por sua vez gera ônus para os cofres públicos e privados (SILVA; FASSA; VALE, 2004).

Portadores de dor lombar tendem a se sentir incapazes de realizar suas atividades de vida diária e frequentemente alimentam forte crença de que toda e qualquer atividade funcional piorará o quadro álgico ou causará algum prejuízo ou limitação física (FRACARO et al., 2013). Em estudo realizado, 32,1\% dos pacientes com lombalgia crônica apresentaram 
quadros de depressão, que pode estar associada a maior intensidade e persistência da dor, maior incapacidade, maior dispêndio econômico e mais eventos adversos de vida (ANTUNES et al., 2013).

O serviço de saúde deste estudo é um Centro de Referência em Saúde do Trabalhador (CEREST), que compóe a Rede Nacional de Atenção Integral à Saúde do Trabalhador (RENAST). Este serviço atende trabalhadores com suspeita de doença relacionada ao trabalho ou que sofram com ameaças a sua integridade física ou mental, de qualquer categoria profissional, registrados ou náo, servidores públicos, autônomos ou mesmo desempregados (BRASIL, 2014b). A Terapia Ocupacional dentro dos Centros de Referência em Saúde do Trabalhador atende trabalhadores com doenças do trabalho, entre outras intervençóes (DALDON; LANCMAN, 2012; ROBINSON; KENNEDY; HARMON, 2011). Para Fuchs e Cassapian (2012), junto aos pacientes com dor lombar crônica, o foco do terapeuta ocupacional deve ser a melhora do desempenho ocupacional desses pacientes, visando a prevençáo de incapacidades, a promoção da funcionalidade e do bem-estar no desempenho das atividades cotidianas, e desenvolvimento de habilidades e capacidades físicas, além da inserção social, com a manutenção e/ou resgate dos papéis ocupacionais. De acordo com Alencar e Terada (2012), orientaçôes posturais podem ser realizadas para se evitar agravos e riscos de surgimento de sintomas nas tarefas do cotidiano de vida, mas são fundamentais também outras intervençóes que auxiliem no enfrentamento da situação, uma vez que a dor e o afastamento do trabalho podem provocar insegurança, medo, sentimento de inutilidade, entre outros. Segundo as autoras, são indicadas atividades expressivas, reflexivas, corporais, em grupos temáticos, entre outras, e intervenções interdisciplinares.

O objetivo deste estudo foi descrever o perfil de trabalhadores com doenças da coluna vertebral (dorsopatias) atendidos em um serviço de Saúde do Trabalhador, da cidade de Santos-SP.

\section{Método}

O estudo é quantitativo e faz parte de um estudo maior intitulado "Aspectos relacionados ao afastamento do trabalho por LER/DORT e outras dorsopatias relacionadas ao trabalho", coordenado pela professora coautora deste artigo, da Universidade Federal de São Paulo (UNIFESP/BS). Foi inicialmente realizada uma análise documental de prontuários abertos de sujeitos atendidos no Centro de Referência em Saúde do Trabalhador (CEREST), da cidade de
Santos-SP, no período de $1^{\circ}$ de janeiro de 2010 a 31 de dezembro de 2011. O levantamento dos dados foi norteado por um roteiro elaborado para registro em planilha Excel, contendo os dados demográficos (como gênero, faixa etária, escolaridade e moradia), dados de profissão, diagnósticos clínicos, sintomas, entre outros que pudessem ser relevantes ao estudo.

Posteriormente, realizou-se o agrupamento dos diagnósticos, conforme a Classificação Internacional de Doenças (CID-10), sendo selecionados para as análises desse estudo os prontuários de sujeitos com diagnósticos clínicos referentes às "outras dorsopatias" (M50 a M54). Também foi realizado como complemento um levantamento sobre os diagnósticos clínicos estabelecidos para "transtornos dos tecidos moles" (M60 a M79), e para "transtornos mentais" (F20 a F41), para possíveis associaçóes e junto aos sujeitos do estudo.

Devido à grande variedade de profissóes, foi utilizado o setor/atividade profissional segundo a CNAE (Classificação Nacional de Atividades Econômicas), que é um instrumento de padronização nacional dos códigos de atividade econômica e dos critérios de enquadramento utilizados pelos diversos órgãos da Administração Tributária do país, e é aplicado a todos os agentes econômicos que estão engajados na produção de bens e serviços (BRASIL, 2014a).

Os dados foram registrados no programa Microsoft Excel e posteriormente analisados no software estatístico $R$ Development Core Team ${ }^{\ominus}$. A fim de se descrever o perfil dos sujeitos do estudo, realizou-se uma análise exploratória dos dados.

A pesquisa foi submetida e aprovada pelo Comitê de Ética em Pesquisa da Universidade Federal de Sáo Paulo - UNIFESP (protocolo n.186.843, data: 11/01/2013) e seguiu todos os procedimentos éticos necessários para a sua execução.

\section{Resultados e Discussão}

Foram analisados 431 prontuários dos sujeitos que foram atendidos no CEREST, no período de janeiro de 2010 a dezembro de 2011. Desses prontuários, $15,77 \%(\mathrm{n}=68)$ dos trabalhadores apresentaram diagnósticos estabelecidos entre M50 e M54, pela Classificação Internacional de Doenças - CID-10.

Alguns dados demográficos dos trabalhadores estão descritos na Tabela 1.

Conforme observado na Tabela 1, 52,9\% dos sujeitos eram do gênero masculino, e a faixa etária mais acometida entre os sujeitos esteve entre 41 e 50 anos de idade. A variável escolaridade infelizmente não pôde ser obtida, por não constar 
Tabela 1. Perfil demográfico dos trabalhadores.

\begin{tabular}{llcc}
\hline \multicolumn{1}{c}{ Variáveis } & $\mathbf{N}$ & $\%$ \\
\hline \multirow{4}{*}{ Gaixnero etária } & Feminino & 32 & $47,1 \%$ \\
& Masculino & 36 & $52,9 \%$ \\
& $21-30$ & 8 & $11,8 \%$ \\
& $31-40$ & 20 & $29,4 \%$ \\
\multirow{5}{*}{ Moradia } & $41-50$ & 24 & $35,3 \%$ \\
& $51-60$ & 16 & $23,5 \%$ \\
& Praia Grande & 6 & $8,8 \%$ \\
& Santos & 42 & $61,8 \%$ \\
Idade & São Vicente & 12 & $17,6 \%$ \\
& Outras cidades & 8 & $11,8 \%$ \\
& Média: 42,3 DP: 9,7 & \\
\hline
\end{tabular}

Tabela 2. Dados sobre o setor de atividade profissional dos sujeitos.

\begin{tabular}{lcc}
\hline \multicolumn{1}{c}{$\begin{array}{c}\text { Setor/Atividade Profissional/ } \\
\text { CNAE }\end{array}$} & N & \% \\
\hline $\begin{array}{l}\text { Alojamento e alimentação } \\
\begin{array}{l}\text { Atividades administrativas e } \\
\text { serviços complementares }\end{array}\end{array}$ & 6 & 9,2 \\
$\begin{array}{l}\text { Atividades financeiras, de seguros } \\
\text { e serviços relacionados }\end{array}$ & 5 & 7,7 \\
Comércio, reparação de veículos & 4 & 6,2 \\
Construção & 3 & 4,6 \\
Eletricidade, gás e outras & 2 & 3,1 \\
utilidades & & \\
Indústrias de transformação & 2 & 3,1 \\
Informação e comunicação & 3 & 4,6 \\
Outras atividades de serviço & 16 & 24,6 \\
Saúde humana e serviços sociais & 2 & 3,1 \\
Serviços domésticos & 7 & 10,8 \\
Transporte, armazenagem e & 9 & 13,8 \\
correio & & \\
Total & $65^{*}$ & 100,0 \\
\hline
\end{tabular}

*n. de dados obtidos $(\mathrm{n}=65$, pois três prontuários não continham essa informação).

no prontuário (dado atualmente já existente na instituição). A principal queixa dos trabalhadores em consulta inicial foi a de lombalgia, com $73,5 \%$ $(\mathrm{n}=50)$ dos casos, e cervicalgia 29,4\% $(\mathrm{n}=20)$, havendo mais de uma queixa entre alguns. $\mathrm{Na}$ literatura, o fator idade é apontado como sendo de risco para as lombalgias, especialmente nas faixas etárias acima de 40 anos (HEDEAGER MOMSEN et al., 2014; PEDROSO et al., 2013; HELFENSTEIN JUNIOR; GOLDEFUN; SIENA, 2010). Em um estudo com trabalhadores submetidos ao programa de Reabilitação Profissional do INSS, a lombalgia foi prevalente em 264 prontuários de segurados afastados do trabalho (ABREU; RIBEIRO, 2010).

Também apresentaram queixas de sintomas dolorosos não relacionados à região de coluna vertebral
$29,4 \%(\mathrm{n}=20)$ dos trabalhadores, sendo que $27,9 \%$ $(\mathrm{n}=19)$ tinham também diagnósticos estabelecidos para transtornos dos tecidos moles (M60 a M79), além de dorsopatias. Vale ressaltar que $8,8 \%(\mathrm{n}=38)$ dos prontuários analisados $(\mathrm{n}=431)$ não constavam um diagnóstico clínico estabelecido. Encontravam-se em consulta inicial em situação de afastamento do trabalho $57,4 \%(\mathrm{n}=39)$.

Entre as doenças mais encontradas neste estudo, estavam: 19,61\% ( $\mathrm{n}=20)$ outros transtornos de discos intervertebrais (M51); 12,75\% ( $\mathrm{n}=13)$ lumbago com ciática (M54.4); 10,78\% ( $\mathrm{n}=11)$ transtornos de discos lombares e de outros discos intervertebrais com radiculopatia (M51.1); 8,82\% (n=9) dorsalgia (M54). A maior prevalência foi M51, com acometimento em discos intervertebrais. A hérnia discal lombar é o diagnóstico mais comum dentre as alteraçôes degenerativas da coluna lombar, acometendo $2 \%$ a $3 \%$ da população. É a principal causa de cirurgia da coluna na população adulta e pode estar associada a algumas tarefas de trabalho, como atividades de exposição a cargas repetidas e vibração prolongada, entre outros fatores (VIALLE et al., 2010). Meziat Filho e Azevedo e Silva (2011) citam em seu estudo que o diagnóstico de dorsalgia (M54) apresentou elevada frequência entre as causas idiopáticas e caracteriza a não especificação do local da dor. Para Krismer e Van Tulder (2007), em aproximadamente $85 \%$ dos casos não é possível obter um diagnóstico anatomopatológico preciso, sendo mais adequado designá-la por dor nas costas idiopática ou inespecífica. Meziat Filho e Azevedo e Silva (2011) citam algumas dores nas costas com alteraçóes anatomopatológicas: M47 - espondilose, M50 - transtornos dos discos intervertebrais, M51 - outros transtornos dos discos intervertebrais, e que podem levar ao desfecho de aposentadoria por invalidez.

As doenças da coluna lombar têm representado uma das principais causas de incapacidade e afastamento das atividades de trabalho por motivo de doença, representando cerca de $60 \%$ a $80 \%$ das doenças que atingem os trabalhadores (CAMPBELL et al., 2013). De acordo com Meziat Filho e Azevedo e Silva (2011), a dor nas costas é uma das principais causas de invalidez e de auxílio-doença no Brasil.

$\mathrm{Na}$ Tabela 2, encontram-se os setores/atividades profissionais mais encontrados entre os sujeitos do estudo, de acordo com a Classificaçáo Nacional de Atividades Econômicas (CNAE).

O setor/atividade de trabalho mais encontrado entre os sujeitos acometidos foi o de "outras atividades de serviço", com 23,5\% ( $\mathrm{n}=16$ ); seguida de transporte, armazenagem e correio, com $13,2 \%(\mathrm{n}=9)$, e serviços domésticos, com 10,3\% (n=7). Entre as profissóes 
mais prevalentes dos sujeitos estavam: faxineiro $(n=6)$, ajudante geral $(n=3)$ e cozinheiro $(n=3)$. Outras profissóes, como auxiliar de limpeza, auxiliar de enfermagem, carteiro, diarista, doméstica, motorista, operador de caixa, pedreiro, operador de máquina, mecânico de manutenção, entre outras, também apareceram em menor quantidade. Para Leal (2010), há evidências indicando associação moderada entre afecçôes lombares e trabalhos físicos pesados. Para este autor, as maiores incidências de afecções lombares são encontradas na indústria do comércio atacadista, fabricação e construção, setor de serviços, e setor de finanças e seguros. As categorias profissionais que se destacaram neste estudo se relacionam às profissóes com supostas exigências físicas no trabalho.

Dos trabalhadores ( $\mathrm{n}=68), 13,2 \%$ ( $\mathrm{n}=9)$ também apresentaram transtornos mentais, entre F20.9 e F41 pela CID-10. Desses trabalhadores com transtornos mentais ( $n=9), 33,3 \%(n=3)$ eram do gênero masculino, e 66,7\% (n=6) do gênero feminino. Os diagnósticos clínicos mais prevalentes foram: $14,29 \%(\mathrm{n}=2) \mathrm{com}$ F41.0 (Transtorno do pânico), 14,29\% ( $\mathrm{n}=2) \mathrm{com}$ F41.2 (Transtorno misto ansioso e depressivo), $14,29 \%(\mathrm{n}=2)$ com F43.0 (Reação aguda ao estresse), 14,29\% ( $\mathrm{n}=2$ ) com F33.1 (Transtorno depressivo recorrente), 14,29\% ( $\mathrm{n}=2)$ com F32.0 (Episódio depressivo leve), alguns com mais de um.

Pode haver relações entre as doenças lombares e transtornos mentais, em especial a depressão. A dor lombar crônica impede, muitas vezes, a execução satisfatória de atividades de vida diária, de trabalho e até de lazer, fazendo com que os sujeitos acometidos se sintam com medo e parem de realizar suas atividades; quadro propício para o desenvolvimento de depressão e outros transtornos mentais (FRACARO et al., 2013). De acordo com Amaral et al. (2010), pessoas que sofrem de dor crônica na coluna podem acabar desenvolvendo sintomas depressivos, uma vez que sentem dores constantes e incapacitantes, e alteraçóes psicológicas pioram o quadro de dor e restrição física do paciente. Pacientes com lombalgia crônica estão sujeitos a quadros de depressão associados a maior intensidade e persistência da dor e maior incapacidade gerada pela doença, comprometendo a realizaçáo de atividades no dia a dia, tanto de trabalho como de lazer (ANTUNES et al., 2013). Segundo Polizelli e Leite (2010), a dor lombar provoca limitações que vão além do aspecto físico, também abrange aspectos psicológicos e sociais que interferem nas atividades do cotidiano, requerendo adaptaçôes até para vestir a roupa e calçar o sapato; provoca a sensação de medo e insegurança para a realização de qualquer tarefa, o que impede uma satisfação pessoal e provoca situaçóes de chateaçáo e esgotamento.

Sugere-se que há uma gravidade adiante da percepçáo de incapacidade decorrente de mais de um diagnóstico clínico/afecção, e que provavelmente dificulta ainda mais as atividades do cotidiano de vida e em diversos aspectos funcionais, que necessitam de maiores investigações. Alguns sujeitos, 27,9\% ( $\mathrm{n}=19)$ acometidos por dorsopatias, também apresentavam diagnósticos clínicos estabelecidos em transtornos dos tecidos moles (M60 a M79), que poderiam estar associados às LER/DORT.

De acordo com Skjutar et al. (2010), pacientes com dor crônica apresentaram limitaçóes no desempenho ocupacional, com restriçóes ocasionadas por barreiras físicas, emocionais e ambientais. Os autores também citam que entre os sujeitos o comportamento perante a dor impedia o engajamento em atividades, havia a falta de conhecimento sobre os mecanismos do adoecimento e como evitá-los, dificuldades em estratégias de como lidar com a dor, entre outros.

\section{Considerações finais}

Os resultados obtidos neste estudo apontaram para uma prevalência de trabalhadores com doenças da coluna vertebral com faixa etária entre 41 e 50 anos de idade, considerada produtiva, sendo a categoria profissional de maior acometimento a de "outras atividades de serviço”, englobando profissóes com exigências físicas em atividades de trabalho. Houve a prevalência de queixa de lombalgia, e mais da metade dos sujeitos do estudo encontravam-se em situaçáo de afastamento do trabalho.

Alguns trabalhadores apresentaram mais de um diagnóstico clínico, englobando transtornos dos tecidos moles e transtornos mentais. $\mathrm{O}$ artigo promove reflexôes sobre o perfil de trabalhadores acometidos por doenças da coluna vertebral, e em especial com lombalgia e em situação de afastamento do trabalho, e com prováveis necessidades de enfrentamento da doença e de situaçóes relacionais, restabelecimento de funçôes no cotidiano de vida, de orientaçôes, entre outros, que váo em geral além dos procedimentos tradicionais da reabilitação física, reforçando a necessidade também de reabilitação psicossocial em alguns casos e de intervençóes interdisciplinares. Futuros estudos devem aprofundar as investigações e questôes abordadas neste estudo.

\section{Referências}

ABREU, A. T. J. B.; RIBEIRO, C. A. B. Prevalência de lombalgia em trabalhadores submetidos ao programa de reabilitação profissional do Instituto Nacional do Seguro 
Social (INSS), São Luís, MA. Acta Fisiátrica, São Paulo, v. 17, n. 4, p. 148-153, 2010.

ALENCAR, M. C. B.; TERADA, T. M. O afastamento do trabalho por afecçóes lombares: repercussóes no cotidiano de vida dos sujeitos. Revista de Terapia Ocupacional da Universidade de São Paulo, São Paulo, v. 23, n. 1, p. 44-51, 2012.

ALMEIDA, I. C. G. B. et al. Prevalência de dor lombar crônica na população da cidade de Salvador. Revista Brasileira de Ortopedia, São Paulo, v. 43, n. 3, p. 96-102, 2008.

AMARAL, V. et al. Prevalência e relação de fatores emocionais e clínicos em pacientes com discopatia degenerativa. Coluna/Columna, São Paulo, v. 9, n. 2, p. 150-156, 2010 .

ANTUNES, R. S. et al. Dor, cinesiofobia e qualidade de vida em pacientes com lombalgia crônica e depressão. Acta Ortopédica Brasileiras, São Paulo, v. 21, n. 1, p. 27-29, 2013. http://dx.doi.org/10.1590/S141378522013000100005 .

BRASIL. Ministério da Previdência Social. Empresa de Tecnologia e Informaçóes da Previdência Social. Anuário Estatístico de Acidentes de Trabalho da Previdência Social. Brasília, 2012. Disponível em: <http://www.previdencia.gov.br/wp-content/uploads/2013/05/AEPS_2012. pdf>. Acesso em: 18 maio 2014.

BRASIL. Ministério da Receita Federal. Receita Federal do Brasil. Apresentação CNAE. Brasília. Disponível em: <http://www.receita.fazenda.gov.br/pessoajuridica/cnaefiscal/txtcnae.htm>. Acesso em: 10 ago. 2014 a.

BRASIL. Secretaria da Saúde do Estado de Sáo Paulo. Rede Nacional de Atenção Integral à Saúde do Trabalhador. CEREST. Disponível em: <http://www.renastonline.org/tags/cerest>. Acesso em: 15 ago. 2014b.

CAMPBELL, P. et al. The influence of employment social support for risk and prognosis in nonspecific back pain: review and critical synthesis. International Archives of Occupational and Environmental Health, Berlin, v. 86, n. 2, p. 119-137, 2013. http://dx.doi.org/10.1007/ s00420-012-0804-2. PMid:22875173.

CARDOSO, A. C.; BATISTA, M. L. L.; SANDOVAL, R. A. Perfil epidemiológico e fatores relacionados à lombalgia em um grupo de costureiras da empresa têxtil Marka da Paz. EFDeportes, Buenos Aires, v. 16, n. 162, 2011. Disponível em: <http://www.efdeportes.com/ efd162/lombalgia-em-um-grupo-de-costureiras.htm>. Acesso em: 13 ago. 2014.

DALDON, M. T. B.; LANCMAN, S. Terapia Ocupacional na vigilância em saúde do trabalhador. Revista de Terapia Ocupacional da Universidade de São Paulo, São Paulo, v. 23, n. 3, p. 216-222, 2012.

EKMAN, M.; JOHNELL, O.; LIDGREN, L. The economic cost of low back pain in Sweden in 2001. Acta Orthopaedica, London, v. 76, n. 2, p. 275-284, 2005. http://dx.doi.org/10.1080/00016470510030698. PMid:16097556.

FERREIRA, G. D. et al. Prevalência de dor nas costas e fatores associados em adultos do Sul do Brasil: estudo de base populacional. Brazilian Journal of Physical Therapy, Sáo Carlos, v. 15, n. 1, p. 31-36, 2011. http://dx.doi. org/10.1590/S1413-35552011005000001.

FRACARO, G. A. et al. Comparison of psycho-social and functional performance variables in a group of chronic low back pain patients. Revista Dor, São Paulo, v. 14, n. 2, p. 119-123, 2013.

FUCHS, M.; CASSAPIAN, M. R. A Terapia Ocupacional e a dor crônica em pacientes de ortopedia e reumatologia. Cadernos de Terapia Ocupacioal da UFSCar, São Carlos, v. 20, n. 1, p. 107-119, 2012.

HAGEN, K. B.; TAMBS, K.; BJERKEDAL, T. A. A prospective cohort study of risk factors for disability retirement because of back pain in general working population. Spine, New York, v. 27, n. 16, p. 1790-1796, 2002. PMid:12195073.

HEDEAGER MOMSEN, A. M. et al. Multiple somatic symptoms in employees participating in a randomized controlled trial associated with sickness absence due to non-specific low back pain. Spine, New York, v. 14, n. 4, p. 373-378, 2014.

HELFENSTEIN JUNIOR, M.; GOLDEFUM, M. A.; SIENA, C. Lombalgia ocupacional. Revista da Associação Médica Brasileira, São Paulo, v. 56, n. 5, p. 583-589, 2010. http://dx.doi.org/10.1590/S010442302010000500022.

KRISMER, M.; VAN TULDER, M. Low Back Pain Group of the Bone and Joint Health Strategies for Europe Project. Strategies for prevention and management of musculoskeletal conditions. Low back pain (non-specific). Best Practice \& Research. Clinical Rheumatology, Amsterdam, v. 21, n. 1, p. 77-91, 2007. http://dx.doi. org/10.1016/j.berh.2006.08.004. PMid:17350545.

LEAL, J. S. Lombalgia: atualização de conceitos. Revista Mineira de Ortopedia e Traumatologia, Belo Horizonte, v. 1, n. 2, p. 8-18, 2010

LIMA, A. C. et al. Prevalência e características da (in) capacidade para o trabalho de trabalhadores de enfermagem. Journal of Nursing UFPE, Recife, v. 7, n. 8, p. 5143-5149, 2013.

MASCARENHA, F. A. N.; BARBOSA-BRANCO, A. Incapacidade laboral entre trabalhadores do ramo Correios: incidência, duração e despesa previdenciária em 2008. Cadernos de Saúde Pública, Rio de Janeiro, v. 30, n. 6, p. 1315-1326, 2014.

MEZIAT FILHO, N.; AZEVEDO E SILVA, G. Invalidez por dor nas costas entre segurados da Previdência Social do Brasil. Revista de Saúde Pública, São Paulo, v. 45, n. 3, p. 494-502, 2011. 
PEDROSO, A. A. S. et al. Índice de incapacitação das lombalgias em motoristas de caminhão. ABCS Health Sciences, Santo André, v. 38, n. 3, p. 142-145, 2013.

POLIZELLI, K. M.; LEITE, S. N. Quem sente é a gente, mas é preciso relevar: a lombalgia na vida das trabalhadoras do setor têxtil de Blumenau - Santa Catarina. Saúde Sociedade, São Paulo, v. 19, n. 2, p. 405-417, 2010.

ROBINSON, K.; KENNEDY, N.; HARMON, D. Review of occupational therapy for people with chronic pain. Australian Occupational Therapy Journal, Melbourne, v. 58, n. 2, p. 74-81, 2011. http:// dx.doi.org/10.1111/j.1440-1630.2010.00889.x. PMid:21418229.

SILVA, M. C.; FASSA, A. C.; VALLE, N. C. J. Dor lombar crônica em uma populaçáo adulta do Sul do Bra- sil: prevalência e fatores associados. Cadernos de Saúde Pública, Rio de Janeiro, v. 20, n. 2, p. 377-385, 2004.

SKJUTAR, A. et al. Indicators of need for occupational therapy in patients with chronic pain: occupational therapists' focus group. Occupational Therapy International, London, v. 17, n. 2, p. 93-103, 2010. PMid:19653210.

TAVARES NETO, A. et al. Lombalgia na atividade policial militar: análise da prevalência, repercussóes laborativas e custo indireto. Revista Baiana de Saúde Pública, Salvador, v. 37, n. 2, p. 365-374, 2013.

VIALLE, L. R. et al. Hérnia discal lombar. Revista Brasileira de Ortopedia, São Paulo, v. 45, n. 1, p. 17-22, 2010. http://dx.doi.org/10.1590/S010236162010000100004 .

\section{Contribuição dos Autores}

Janaina, Karoline e Maria do Carmo trabalharam na concepção do artigo. Karoline participou do trabalho de campo, com a colaboração da Dra. Lucy e Janaina. Maria do Carmo realizou o trabalho de orientação e supervisão do campo e estudo. Todos autores aprovaram a versão final do texto.

\section{Fonte de Financiamento}

Fundação de Amparo à Pesquisa do Estado de São Paulo - FAPESP. 